V. A. Galaktionov

\title{
On higher-order semilinear parabolic equations with measures as initial data
}

Received March 3, 2003

Abstract. We consider $2 m$ th-order $(m \geq 2)$ semilinear parabolic equations

$$
u_{t}=-(-\Delta)^{m} u \pm|u|^{p-1} u \quad \text { in } \mathbb{R}^{N} \times \mathbb{R}_{+}(p>1),
$$

with Dirac's mass $\delta(x)$ as the initial function. We show that for $p<p_{0}=1+2 m / N$, the Cauchy problem admits a solution $u(x, t)$ which is bounded and smooth for small $t>0$, while for $p \geq$ $p_{0}$ such a local in time solution does not exist. This leads to a boundary layer phenomenon in constructing a proper solution via regular approximations.

Keywords. Cauchy problem, semilinear parabolic equation, Dirac mass, existence, nonexistence

\section{Introduction: Brezis-Friedman results and higher-order equations}

\subsection{Semilinear second-order heat equation}

In 1983, H. Brezis and A. Friedman [3] studied the semilinear heat equation with a measure in the initial condition

$$
\begin{array}{ll}
u_{t}=\Delta u-|u|^{p-1} u & \text { in } Q=\mathbb{R}^{N} \times \mathbb{R}_{+}, \\
u(x, 0)=\delta(x) & \text { in } \mathbb{R}^{N},
\end{array}
$$

where $p>0$ is a fixed exponent and $\delta(x)$ is the Dirac mass at 0 (the Dirichlet problem in a bounded domain $\Omega$ containing 0 was included). It was established that there exists the critical exponent (same as Fujita's exponent for reaction-diffusion equations)

$$
p_{0}=1+2 / N
$$

such that the following results hold.

Research supported by RTN network HPRN-CT-2002-00274.

V. A. Galaktionov: Department of Mathematical Sciences, University of Bath, Bath BA2 7AY, UK and Keldysh Institute of Applied Mathematics, Miusskaya Sq. 4, 125047 Moscow, Russia; e-mail: vag@maths.bath.ac.uk

Mathematics Subject Classification (2000): 35K55, 35K65 
(I) Existence ([3, Theorem 3]): in the subcritical range $p<p_{0}$, there exists a unique solution $u \in C^{2,1}(Q) \cap L^{p}(Q)$ satisfying 1.1 in the sense of distributions and the initial condition 1.2 in the weak topology of measures,

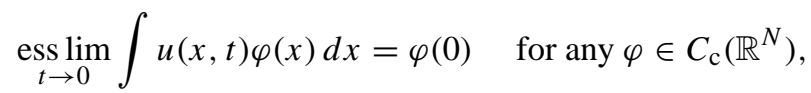

where $C_{\mathrm{c}}\left(\mathbb{R}^{N}\right)$ is the space of all continuous compactly supported functions in $\mathbb{R}^{N}$.

(II) Nonexistence ([3, Theorem 1]): for $p \geq p_{0}$, the Cauchy problem does not have a solution $u(x, t) \in L_{\text {loc }}^{p}(Q)$ satisfying equation 1.1 and $[1.4$. Moreover, any suitable regular approximation $\left\{u_{j}(x, t)\right\}$ via classical bounded solutions of 11.1 with bounded initial data $u_{0 j}$ converging to $\delta$ gives the trivial limit ([3, Theorem 4]):

$$
u_{j}(x, t) \rightarrow 0 \quad \text { as } j \rightarrow \infty \text { uniformly on } \mathbb{R}^{N} \times[\varepsilon, T] \text { for any small } \varepsilon>0 .
$$

The paper [3] was the first to treat remarkable properties of nonlinear parabolic equations with measures as initial functions (earlier references on elliptic problems for $-\Delta u+$ $|u|^{p-1} u=f$, a measure, are presented therein) and established the crucial fact that for a class of "supercritical" nonlinearities, parabolic equations cannot admit measures as initial functions.

Convergence (1.5) for $p \geq p_{0}$ means that a boundary layer occurs at $t=0$ [3] , and that $\bar{u}(x, t) \equiv 0$ for $t>0$ can be treated as a unique (proper) solution of the Cauchy problem (1.1), (1.2). This was a remarkable example showing that the "extended" semigroup constructed by regular approximations is essentially discontinuous at $t=0$ for initial bounded measures from $M\left(\mathbb{R}^{N}\right)$. Later on, it was understood in the theory of blow-up and extinction phenomena that semigroups induced by various nonlinear parabolic equations of reaction-diffusion-absorption kind cannot be continuous in general, and singularities occurring at finite $t=T>0(T=0$ implies initial singularity as above) can create unavoidable discontinuities in any weak topology; see [2], [11] and references therein.

\subsection{Extension to higher-order semilinear parabolic equations}

As a key example, we consider the Cauchy problems for the $2 m$ th-order semilinear parabolic equation

$$
u_{t}=-(-\Delta)^{m} u+g(u) \quad \text { in } Q, \quad u(x, 0)=\delta(x) \quad \text { in } \mathbb{R}^{N},
$$

where the nonlinear term $g(u)$ has algebraic homogeneity $p>1$,

$$
g(u)= \pm|u|^{p-1} u \quad \text { or } \quad g(u)= \pm|u|^{p} .
$$

Our main goal is to show that the local existence (Section 3) and nonexistence results (Section 4) of type (I) and (II) remain true for higher-order equations with the critical exponent depending on $m$,

$$
p_{0}=1+2 m / N \text {. }
$$

In Section 5, for $p \geq p_{0}$, we discuss the structure of the boundary layer at $t=0$ giving a natural proper solution in this nonexistence range. 
It is important that $(1.8)$ is a crucial exponent in other problems for higher-order semilinear parabolic equations. For instance, it is Fujita's one for

$$
u_{t}=-(-\Delta)^{m} u+|u|^{p} \quad \text { in } Q, \quad p>1 .
$$

Namely, if $p \leq p_{0}$, then for arbitrarily small initial data with nonnegative first Fourier coefficient, $\int u_{0} \geq 0$, the solution $u(x, t) \not \equiv 0$ blows up in finite time, while for $p>p_{0}$ all small solutions are global; see [5], [6], [9] and references therein.

Similar critical exponents occur in local-in-time existence theory. We refer to F. B. Weissler's results [14], [15] who first showed existence of critical exponents for solvability of higher-order semilinear equations in $L^{q}$. For instance, for any $m \geq 1$, local existence and uniqueness of an $L^{q}$-solution with initial data in $L^{q}(q>1)$ for equations 1.9 was proved to hold if $p<p_{q}=1+2 m q / N$ (observe that $p_{q}=p_{0}$ for $q=1$ ).

There exists a principal difference between local solvability for data in $L^{1}\left(\mathbb{R}^{N}\right)$ and measures in $M\left(\mathbb{R}^{N}\right)$. Namely, problem 1.6 does not have a local solution for any nonlinearity in 1.7 with $p \geq p_{0}$, while for $u_{0} \in L^{1}$ there exists a local (and global) solution in the monotone case $g(u)=-|u|^{p-1} u$ with any $p>0$ (cf. [3, p. 83] for $m=1$ ). Concerning global existence, in the supercritical range $p>p_{0}$, solutions of (1.9) are global for any sufficiently small initial data; see various approaches in [1], [4], [6] and [9].

\section{Preliminaries}

\subsection{Fundamental solution}

For any $m \geq 1$, the fundamental solution $b(x, t)$ of the linear parabolic equation

$$
u_{t}=-(-\Delta)^{m} u \quad \text { in } Q
$$

has the self-similar form

$$
b(x, t)=t^{-N / 2 m} f(y), \quad y=x / t^{1 / 2 m},
$$

where $f$ is a unique radial solution of the elliptic equation

$$
\begin{gathered}
\mathbf{B} f \equiv-(-\Delta)^{m} f+\frac{1}{2 m} \nabla f \cdot y+\frac{N}{2 m} f=0 \quad \text { in } \mathbb{R}^{N}, \quad \int_{\mathbb{R}^{N}} f=1, \\
f(y)=(2 \pi)^{-N / 2} \int_{0}^{\infty} e^{-s^{2 m}}(s|y|)^{N / 2} J_{(N-2) / 2}(s|y|) d s,
\end{gathered}
$$

where $J_{v}$ denotes Bessel's function. The rescaled kernel $f$ satisfies the estimate [7]

$$
|f(y)|<D F(y) \equiv D \omega_{1} e^{-d|y|^{\alpha}} \quad \text { in } \mathbb{R}^{N}, \quad \alpha=2 m /(2 m-1) \in(1,2),
$$

where $D>1$ and $d>0$ are constants depending on $m$ and $N$ and $\omega_{1}>0$ is a normalization factor such that $\int F=1$. Note that

$$
\bar{b}(x, t)=t^{-N / 2 m} F\left(x / t^{1 / 2 m}\right)>0 \quad \text { in } Q
$$


is the majorizing kernel of the majorizing order-preserving integral equation. The constant $D>1$ for $m>1$ is the order deficiency of the linear operator $(D=1$ if and only if the linear semigroup is order-preserving, which occurs for $m=1$ only) [9].

As a standard practice, given initial data $u_{0} \in L^{q}\left(\mathbb{R}^{N}\right)$ with some $q \geq 1$, the Cauchy problem can be studied via the corresponding integral equation

$$
u(t)=b(t) * u_{0}+\int_{0}^{t} b(t-s) * g(u(s)) d s \quad \text { for } t>0
$$

where a solution can be understood as a curve $u \in C\left([0, T] ; L^{q}\right) \cap C^{1}\left((0, T] ; L^{q}\right)$ or as a weaker mild solution (see [15], [4] and [1]). Local solvability of such equations is proved by contraction mapping arguments (see e.g. [15] and references to Chapter 15 in [13]). In view of blowing up solutions for [1.9] or for $g(u)=|u|^{p-1} u$ (see [5] and [9]), in these cases the solutions can be essentially local in time. Global existence for $g(u)=-|u|^{p-1} u$ is associated with a priori bounds via the monotonicity and coercivity of the operator on the right-hand side of (1.1).

\subsection{Eidel'man's estimate on iterated kernels}

We formulate a useful estimate on iterated majorizing kernels 2.6 from S. D. Eidel'man's book [7, Lemma 5.1 on pp. 35-36]. See details in [6] where such estimates are used for proving global existence results for higher-order semilinear parabolic equations.

Proposition 2.1. For the integral

$$
I_{1}=\int_{\mathbb{R}^{N}}[(t-\beta)(\beta-\tau)]^{-N / 2 m} \exp \left\{-d\left[\frac{|x-y|^{\alpha}}{(t-\beta)^{\alpha / 2 m}}+\frac{|y-\xi|^{\alpha}}{(\beta-\tau)^{\alpha / 2 m}}\right]\right\} d y,
$$

where $\tau<\beta<t$, the following estimate holds:

$$
I_{1} \leq M(\varepsilon)(t-\tau)^{-N / 2 m} \exp \left\{-(d-\varepsilon) \frac{|x-\xi|^{\alpha}}{(t-\tau)^{\alpha / 2 m}}\right\}
$$

$\varepsilon \in(0, d)$ being arbitrary, $M(\varepsilon)=M_{1} \varepsilon^{-N / \alpha}$ and $M_{1}>0$ is independent of $\varepsilon$.

\section{Existence for $p<p_{0}$}

We mention again that local existence for initial data $u_{0} \in L^{q}$ with $q>1$ is known for a long period; see Theorem 2 in [15] (the restriction $p<1+2 m q / N$ serves the noncoercive case of equations like (1.9)). Various extensions (including $L^{1}$-data) can be found in [4] and [1]. In the second-order case $m=1$, a solution of (1.1), 1.2 was known to exist for all $p>0[3]$. 


\subsection{Existence theorem}

For $m>1$ and measures as initial data, we apply a contraction mapping argument to ensure existence of a bounded classical (i.e., smooth as the regularity of $|u|^{p-1} u$ or $|u|^{p}$ dictates) solution for $t>0$ of the integral equation (2.7) with $u_{0}=\delta$, so that $b(t) * \delta=$ $b(t)$,

$$
u(t)=\mathbf{M}(u)(t) \equiv b(t)+\int_{0}^{t} b(t-s) * g(u(s)) d s \quad \text { for } t>0 .
$$

We will study $(3.1)$ in a weighted space of continuous functions. Fix a $v>0$ and define $Q_{v}=\mathbb{R}^{N} \times(0, v]$. The weight function is associated with the majorizing rescaled kernel $F$ in 2.5 ,

$$
\rho(x, t)=t^{-N / 2 m} \exp \left\{-k|x|^{\alpha} / t^{\alpha / 2 m}\right\}>0 \quad \text { for } t>0, \text { where } k=d / p .
$$

In the Banach space $C_{\rho}\left(Q_{\nu}\right)$ of functions $u$ such that $v=\frac{1}{\rho} u \in C\left(Q_{\nu}\right)$ with the sup-norm

$$
\|u\|_{\rho}=\sup _{Q_{v}}|v(x, t)|
$$

we consider a closed subset

$$
Y_{A}=\left\{u \in C_{\rho}\left(Q_{\nu}\right):\|u\|_{\rho} \leq A\right\} \quad \text { with a positive constant } A .
$$

Theorem 3.1. Let $p \in\left(1, p_{0}\right)$. Then for any $A>D \omega_{1}$, there exists a sufficiently small $v>0$ such that equation (3.1) has a unique solution $u \in Y_{A}$ which is classical in $Q_{v}$ and satisfies (1.4).

The theorem establishes existence and uniqueness of the solution in an arbitrarily large "neighbourhood" of the fundamental solution for $t \approx 0$, with neighbourhoods defined via the positive majorizing kernel (2.6). Then $u(x, t)$ is classical for $t \in(0, v]$, and its further continuation depends on the nonlinearity $g(u)$. For instance, in the monotone case $g(u)=-|u|^{p-1} u$ the solution is supposed to be bounded and global (at least for $p<$ $\left.p_{\text {Sob }}=(N+2 m) /(N-2 m)\right)$, while for $g(u)=+|u|^{p-1} u$ it may blow up in finite time.

Proof. We apply Banach's Contraction Principle (see [13]).

(i) Let us show that $\mathbf{M}: Y_{A} \rightarrow Y_{A}$ if $v \ll 1$. Given a $u \in Y_{A}$, from 3.1 we have

$$
|\mathbf{M}(u)| \leq|b(t)|+\left|\int_{0}^{t}(\cdot) d s\right| \equiv J_{1}+J_{2} .
$$

It follows from (2.5) that since $d=p k>k$,

$$
J_{1} \leq D \omega_{1} \rho(x, t) .
$$

Estimating the second term $J_{2}$ for $u \in Y_{A}$, from (3.4, 2.5 and $d=k p$ we have

$$
J_{2} \leq C_{0} \int_{0}^{t} s^{-N(p-1) / 2 m}[(t-s) s]^{-N / 2 m} \int \exp \left\{-d\left[\frac{|x-y|^{\alpha}}{(t-s)^{\alpha / 2 m}}+\frac{|y|^{\alpha}}{s^{\alpha / 2 m}}\right]\right\} d y d s,
$$


where $C_{0}=D \omega_{1} A^{p}$. We put $\xi=0, \beta=s, \tau=0$ and

$$
d-\varepsilon=d / p=k, \quad \text { so that } \quad \varepsilon=d(p-1) / p,
$$

in Proposition 2.1 to get

$$
J_{2} \leq C_{0} M(\varepsilon)\left(\int_{0}^{t} s^{-N(p-1) / 2 m} d s\right) \rho(x, t) \leq C_{0} M(\varepsilon) \mu^{-1} \nu^{\mu} \rho(x, t),
$$

where $\mu=N\left(p_{0}-p\right) / 2 m>0$. By estimates 3.6) and 3.7), for any $u \in Y_{A}$ we obtain

$$
|\mathbf{M}(u)| \leq D \omega_{1}\left(1+M(\varepsilon) A^{p} \mu^{-1} v^{\mu}\right) \rho(x, t),
$$

so that $\mathbf{M}(u) \in Y_{A}$ provided that

$$
D \omega_{1}\left(1+M(\varepsilon) A^{p} \mu^{-1} \nu^{\mu}\right) \leq A,
$$

i.e., for any fixed $A>D \omega_{1}$ if $v>0$ is sufficiently small.

(ii) We now show that $\mathbf{M}$ is a contraction in $Y_{A}$ provided that $v \ll 1$. Taking any $u_{1}, u_{2} \in Y_{A}$ and estimating the difference $\left|u_{1}\right|^{p-1} u_{1}-\left|u_{2}\right|^{p-1} u_{2}$ by using Lagrange's formula of finite increments yields

$$
\begin{aligned}
& \left\|\mathbf{M}\left(u_{1}\right)-\mathbf{M}\left(u_{2}\right)\right\|_{\rho}=\sup \frac{1}{\rho(t)}\left|\int_{0}^{t} b(t-s) *\left(\left|u_{1}\right|^{p-1} u_{1}-\left|u_{2}\right|^{p-1} u_{2}\right)(s) d s\right| \\
& \leq \sup \frac{1}{\rho(t)} \int_{0}^{t}|b(t-s)| * \rho(s) \frac{1}{\rho(s)}\left|u_{1}(s)-u_{2}(s)\right| p \int_{0}^{1}\left(z\left|u_{1}\right|+(1-z)\left|u_{2}\right|\right)^{p-1} d z d s .
\end{aligned}
$$

Therefore, since $\left|u_{1,2}\right| \leq A \rho$ we obtain

$$
\left\|\mathbf{M}\left(u_{1}\right)-\mathbf{M}\left(u_{2}\right)\right\|_{\rho} \leq J_{3}\left\|u_{1}-u_{2}\right\|_{\rho},
$$

where

$$
J_{3}=C_{1} \sup \frac{1}{\rho(t)} \int_{0}^{t}|b(t-s)| * \rho^{p}(s) d s, \quad C_{1}=p D \omega_{1} A^{p-1} .
$$

By Proposition 2.1.

$$
\begin{aligned}
J_{3} \leq C_{1} \sup \frac{1}{\rho(t)} \int_{0}^{t} s^{-N(p-1) / 2 m}[(t-s) s]^{-N / 2 m} \int & \exp \left\{-d \frac{|x-y|^{\alpha}}{\left.(t-s)^{\alpha / 2 m}\right\}}\right. \\
& \times \exp \left\{-d \frac{|y|^{\alpha}}{s^{\alpha / 2 m}}\right\} d y d s \\
\leq C_{1} M(\varepsilon) \sup \frac{1}{\rho(t)}\left(\int_{0}^{t} s^{-N(p-1) / 2 m} d s\right) \rho(t), &
\end{aligned}
$$

with $\varepsilon$ from 3.8 , so that $J_{3} \leq C_{1} M(\varepsilon) \mu^{-1} v^{\mu}<1$ for any small $v>0$.

Thus, there exists a unique fixed point of the operator $\mathbf{M}$ in $Y_{A}$. Since $u(x, t)$ is bounded for any $t>0$, it is a classical solution of the parabolic PDE. Using estimate 3.9 in passing to the limit $t \rightarrow 0^{+}$in equation 3.1, we obtain 1.4. 
Corollary 3.2. The solution constructed in Theorem 3.1 satisfies the initial condition (1.2) in the following rescaled sense: as $t \rightarrow 0$,

$$
t^{N / 2 m} u\left(y t^{1 / 2 m}, t\right) \rightarrow f(y) \quad \text { uniformly in } y \in \mathbb{R}^{N} .
$$

This follows from estimate (3.9). As another straightforward consequence, it follows that this unique solution can be constructed by regular approximations.

Corollary 3.3. Let $u_{0 j} \rightarrow \delta$ in the topology of bounded measures be a sequence of initial functions from $L^{\infty}\left(\mathbb{R}^{N}\right) \cap L^{1}\left(\mathbb{R}^{N}\right)$ such that the corresponding sequence $\left\{u_{j}(x, t)\right\}$ of classical solutions of 1.1 in $\bar{Q}_{v}$ is contained in $Y_{A}$. Then

$$
u_{j} \rightarrow u \quad \text { uniformly on compact subsets of } Q_{\nu},
$$

where $u$ is the unique solution of 3.1.

Proof. Compactness of the subset $\left\{u_{j}\right\}$ in $C$ on compact subsets of $Q_{v}$ follows from uniform estimates quite similar to 3.10 on classical solutions of the PDE for $t>0$ [7], passage to the limit $j \rightarrow \infty$ in the integral equations for $u_{j}$ is straightforward, and then convergence (3.14) along the whole sequence is guaranteed by the uniqueness of the solution $u \in Y_{A}$.

\subsection{More general nonlinearities}

By Theorem 3.1, the existence of a classical solution is associated with the convergence of the integral in 3.9 , and $p=p_{0}$ is the minimal critical exponent where the integral diverges. We can improve this existence result for equation (1.6) by including a more general nonlinearity $g: \mathbb{R} \rightarrow \mathbb{R}$, which is a continuous, increasing, odd function. In view of the existence theorem, it is important to study $g(u)$ close for $u \gg 1$ to the critical behaviour with the exponent $p=1+2 m / N$ by setting

$$
g(u)= \pm \kappa(u)|u|^{2 m / N} u,
$$

with a positive, slowly varying as $u \rightarrow \infty$ (e.g., logarithmically, see below), even, continuous and monotone function $\kappa: \mathbb{R} \rightarrow \mathbb{R}_{+}$. We first present a typical existence result for a particular logarithmic factor.

Theorem 3.4. Let

$$
\kappa(u)=\ln ^{\beta}(e+|u|) \quad(\beta<0) .
$$

Then for any $\beta<-1$, equation (3.1), 3.15) has a bounded solution $u(x, t)$ for small $t>0$ satisfying (1.4).

Proof. We need a slight modification of the proof of Theorem 3.1 Namely, we fix a sufficiently small constant $\mu>0$ and set $k=(d+\mu) / p$ in 3.2 . Then in both estimates (3.9) and 3.12 we establish an upper bound of the term $\kappa(A \rho(y, s))$, which is done as follows: for the given $\mu>0$, there exists a constant $C_{\mu}>0$ such that

$$
\left|\kappa\left(A s^{-N / 2 m} \exp \left\{-k|y|^{\alpha} / s^{\alpha / 2 m}\right\}\right)\right| \leq \kappa\left(s^{-N / 2 m}\right) C_{\mu} \exp \left\{\mu|y|^{\alpha} / s^{\alpha / 2 m}\right\} .
$$


This is easy to check for the nonlinearity 3.16 for any $\beta<0$. Then we arrive at estimate $3.9), \varepsilon=(d(p-1)-\mu) / p$, with the integral replaced by $\int_{0}^{t} \kappa\left(s^{-N / 2 m}\right) d s / s$, which converges iff $\beta<-1$. The same converging integral occurs in 3.12.

Similarly, we prove that a bounded solution exists for

$$
\kappa(u)=\ln ^{\beta}(\ln u) / \ln u \quad \text { for } u \gg 1,
$$

provided that $\beta<-1$. For a general decreasing positive perturbation factor $\kappa(u) \leq 1$ in (3.15), the existence analysis leads to the following Osgood-like criterion (cf. the corresponding nonexistence result in the next section):

$$
\int^{\infty} \kappa\left(e^{N z / 2 m}\right) d z<\infty .
$$

In order to obtain an estimate like (3.17) we need an extra technical assumption. Namely, fixing an arbitrarily small $\mu>0$, setting $a=s^{-N / 2 m} \rightarrow \infty$ as $s \rightarrow 0$ (dependence on $A$ is not essential) and $z=k|y|^{\alpha} / s^{\alpha / 2 m}$, we find that for decreasing $\kappa$, for a fixed $a>0$,

$$
\kappa\left(a e^{-z}\right) \leq C_{\mu}(a) e^{\mu z} \text { for all } z \geq 0,
$$

where $C_{\mu}(a)$ comes from the conditions $C_{\mu}(a) \geq 2 \kappa(a)$ and $C_{\mu}(a) e^{\mu z_{a}} \geq 1$ with $z_{a}$ determined from the equation $\kappa\left(a e^{-z}\right)=2 \kappa(a)$, i.e., $C_{\mu}(a)=\max \left\{2 \kappa(a), e^{-\mu z_{a}}\right\}$. Then the boundedness of $C_{\mu}(a)$,

$$
\left.C_{\mu}(a) \leq \text { const } \quad \text { as } a \rightarrow \infty \text { (i.e., } s \rightarrow 0\right),
$$

guarantees that estimate 3.20 holds for all sufficiently large $a=s^{-N / 2 m}$. Using the same proof, we arrive at the criterion (3.19). The functional inequality (3.21) (which is obviously true for factors 3.16 and 3.18 ) holds for most of the typical nonlinearities of interest.

\section{Nonexistence for $p \geq p_{0}$}

Looking for a solution of 3.1 with $p \geq p_{0}$ satisfying $u \in L_{\mathrm{loc}}^{p}(Q)$, we have

$$
\begin{gathered}
\|u(t)-b(t)\|_{1} \leq \int_{0}^{t} \int|u|^{p}(s) d s \rightarrow 0 \quad \text { for a.e. } t \rightarrow 0, \quad \text { i.e., } \\
u(t)=b(t)+o(1) \quad \text { in } L^{1}\left(\mathbb{R}^{N}\right) \text { for a.e. } t \approx 0^{+} .
\end{gathered}
$$

This asymptotic behaviour as $t \rightarrow 0$ is not sufficient to guarantee nonexistence of such a solution. Bearing in mind the existence theorem in Section 4 dealing with sufficiently regular local solutions, we formulate a straightforward version of the nonexistence result in terms of the asymptotic behaviour typically exhibited by the classical solutions. Nevertheless, in our opinion, it correctly reflects the "essence" of nonexistence.

Theorem 4.1. For $p \geq p_{0}, 3.1$ does not have a solution $u \in L_{\mathrm{loc}}^{p}(Q)$ satisfying 3.13 , uniformly on any arbitrarily small compact subset in $y$. 
Actually, assumption like 3.13 means that the first term on the right-hand side of 2.7] (or the PDE (1.6) determines the asymptotic behaviour as $t \rightarrow 0$ in the pointwise rescaled sense, and the nonlinear term is negligible there, as happens for any $p<p_{0}$. We easily show that this is not the case for $p \geq p_{0}$.

Proof. Fixing a small subset in $y$, say, a ball $\{|y| \leq r\}$ in 3.13 , we find that for all $t \approx 0$,

$$
\int_{0}^{t} \int|u(s)|^{p} d s \geq C_{1} \int_{0}^{t} s^{N(1-p) / 2 m} d s,
$$

where $C_{1}=\frac{1}{2} \int_{\{|y| \leq r\}}|f(y)|^{p} d y>0$. The integral on the right-hand side diverges iff $N(1-p) / 2 N \leq-1$, i.e., for $p \geq p_{0}$.

Using the triangle inequality in $L^{p}(Q)$, one can obtain nonexistence under the assumption $u(t)=b(t)+o(1)$ in $L^{p}\left(\mathbb{R}^{N}\right)$ for a.e. $t \approx 0^{+}$. It is interesting to establish nonexistence under weaker assumptions as in [3] for $m=1$. Note that such generalizations to $m \geq 2$ are expected to be very hard, as usually happens in translating delicate asymptotic results to nonlinear higher-order equations. Some of the key techniques of [3] apply only to the second-order equations admitting a number of order-preserving, comparison and contractivity properties related to the Maximum Principle. For instance, multiplying the equation by approximation of $\operatorname{sign}(u)$ (or of $\operatorname{sign}(u-v)$ for the difference of two solutions $u$ and $v$ ) and integrating by parts giving crucial estimates for the $L^{1}$-theory do not apply to $2 m$ th-order parabolic operators. The same happens to Kato's inequalities and some other techniques.

Remark (on general nonlinearities). For equations (1.6) or (3.1) with a general nonlinearity $g(u)$, for initial measure $(1.2)$, a solution (in the same sense as above) such that $g(u) \in L_{\mathrm{loc}}^{1}\left(Q_{v}\right)$ does not exist if

$$
g(b) \notin L_{\text {loc }}^{1}\left(Q_{\nu}\right) .
$$

Consider the nonlinear term (3.15) with the same assumptions on $\kappa$. Then 4.3 leads to the following nonexistence Osgood criterion on $\kappa$ (cf. the existence criterion (3.19)):

$$
\int^{\infty} \kappa\left(e^{N z / 2 m}\right) d z=\infty .
$$

In both logarithmic cases 3.16 and 3.18 the nonexistence occurs in the range $\beta \geq-1$, which is complementary to the existence range $\beta<-1$ in Theorem 3.4. For the general scaling invariant nonlinearity

$$
g(u)=|u|^{p_{0}-1} u|D u|^{p_{1}}\left|D^{2} u\right|^{p_{2}} \cdots\left|D^{2 m-1} u\right|^{p_{2 m-1}},
$$

where $\left|D^{k} u\right|$ denotes the length of the vector $\left\{D^{\beta} u:|\beta|=k\right\}$ and $p_{k} \geq 1$ are given exponents, the nonexistence condition 4.3 holds if $\sum p_{k}(N+k) \geq 2 m+N$. Local existence results in $L^{p}$ for some nonlinearities like that can be found in [4].

Main local existence/nonexistence results apply to more general semilinear higherorder parabolic equations with linear operators $\sum_{|\beta|<2 m} a_{\beta}(x) D^{\beta} u$ having real bounded coefficients $a_{\beta}$ provided that suitable estimates like $[2.5)$ on the rescaled kernel are available (see [7], [12]). 


\section{On formation of boundary layers at $t=0$ for $p \geq p_{0}$}

In view of nonexistence of a solution continuous at $t=0$, it was pointed out in [3, Section 4] that this leads to a boundary layer phenomenon. We now describe some common aspects of formation of boundary layers which apply for any $m \geq 1$. Dealing with the Cauchy problem (1.6), we choose a natural regular approximation

$$
b(\varepsilon) \rightarrow \delta \quad \text { as } \varepsilon \rightarrow 0^{+}
$$

of Dirac's mass. By $\left\{u_{\varepsilon}(t)\right\}$ we denote the corresponding family of local in time classical solutions of $(1.6)$ with initial data $b(\varepsilon)$. Next we will study the behaviour of $u_{\varepsilon}(t)$ as $\varepsilon \rightarrow 0^{+}$which is expected to determine a suitable proper solution $\bar{u}(t)$ in this case of the corresponding extended semigroup being discontinuous at $t=0$.

\subsection{Boundary layer and proper solution in the absorption case}

Let $g(u)=-|u|^{p-1} u$ in the PDE 1.6 . We perform the scaling

$$
u_{\varepsilon}=(t+\varepsilon)^{-N / 2 m} v_{\varepsilon}, \quad y=x /(t+\varepsilon)^{1 / 2 m}
$$

leading to the following equation for the rescaled function $v=v_{\varepsilon}(y, t)$ :

$$
(t+\varepsilon) v_{t}=\mathbf{B} v-(t+\varepsilon)^{-\gamma}|v|^{p-1} v \quad \text { for } t>0, \quad v(y, 0)=f(y),
$$

where the initial function $f$ is the rescaled kernel in 2.2) and $\mathbf{B}$ is the corresponding linear operator 2.3. Note that $\mathbf{B} f=0$. The exponent $\gamma$ in the nonautonomous perturbation in 5.3 is given by

$$
\gamma=N(p-1) / 2 m-1>0 \text { for } p>p_{0} \quad \text { and } \quad \gamma=0 \quad \text { for } p=p_{0} .
$$

Supercritical case $p>p_{0}$. Then $\gamma>0$ in 5.3 so that the perturbation term is unbounded as $\varepsilon \rightarrow 0$ and $t \rightarrow 0$, i.e., it is dominant in the limit. Actually, this is the reason for nonexistence of a continuous solution in this range. A correct prediction of the structure of the boundary layer formed for $t \approx 0$ as $\varepsilon \rightarrow 0$ is described by the ODE obtained by neglecting the linear elliptic operator $\mathbf{B}$ in 5.3 ,

$$
(t+\varepsilon) V_{t}=-(t+\varepsilon)^{-\gamma}|V|^{p-1} V \quad \text { for } t>0, \quad V(y, 0)=f(y) .
$$

Integrating 5.5 yields the explicit expression

$$
V_{\varepsilon}(y, t)=\operatorname{sign} f(y)\left[|f(y)|^{1-p}+(p-1) \gamma^{-1}\left(\varepsilon^{-\gamma}-(t+\varepsilon)^{-\gamma}\right)\right]^{-1 /(p-1)} .
$$

Therefore, for a fixed small $t_{0}>0$ and any $y$ such that $f(y) \neq 0$, we have

$$
V_{\varepsilon}\left(y, t_{0}\right)=\operatorname{sign} f(y) c_{0} \varepsilon^{\gamma /(p-1)}(1+o(1)) \quad \text { as } \varepsilon \rightarrow 0,
$$

where $c_{0}=[(p-1) / \gamma]^{-1 /(p-1)}$, and then 5.2 applies describing the boundary layer.

For the parabolic problem (5.3), introducing the new time variable

$$
s=\gamma^{-1}\left[\varepsilon^{-\gamma}-(t+\varepsilon)^{-\gamma}\right]:\left[0, t_{0}\right] \rightarrow\left[0, s_{0}\right],
$$


we obtain a singular perturbed equation

$$
\begin{gathered}
v_{s}=\psi_{\varepsilon}(s) \mathbf{B} v-|v|^{p-1} v \quad \text { for } s \in\left(0, s_{0}\right], \quad v(y, 0)=f(y), \\
\psi_{\varepsilon}(s)=\varepsilon^{\gamma}\left(1-\gamma \varepsilon^{\gamma} s\right)^{-1},
\end{gathered}
$$

where $\psi_{\varepsilon}(s)=O\left(\varepsilon^{\gamma}\right)$ for sufficiently small $s$ and $\psi_{\varepsilon}\left(s_{0}\right)=O\left(t_{0}^{\gamma}\right)$, i.e., the coefficient of the higher-order derivative is uniformly small on $\left[0, s_{0}\right]$ provided that $t_{0}, \varepsilon \ll 1$. The passage to the limit $\varepsilon \rightarrow 0$ in the singular perturbed equation (5.8) is a hard open problem which we do not study here. We derive a boundary layer estimate under the following assumption:

$$
|v(y, s)| \leq C_{0} s^{-1 /(p-1)} \quad \text { in }\left(0, s_{0}\right] \times \mathbb{R}^{N},
$$

where the constant $C_{0}>0$ is independent of $t_{0}$ and $\varepsilon$. For $m=1$ it is straightforward and follows by comparison with the ODE solution $v^{\prime}=-|v|^{p-1} v$. For any $m>1$, the Maximum Principle is not true and estimates like (5.10) are still not known, representing a challenging open problem. Then the scaling transformation (5.2) yields the following behaviour of the approximating classical solution for small $t_{0}>0$ as $\varepsilon \rightarrow 0$ :

$$
\begin{aligned}
\left|u_{\varepsilon}\left(x, t_{0}\right)\right| & =\left(t_{0}+\varepsilon\right)^{-N / 2 m}\left|v\left(y, s_{0}\right)\right| \leq\left(t_{0}+\varepsilon\right)^{-N / 2 m} C_{0} s_{0}^{-1 /(p-1)} \\
& =t_{0}^{-N / 2 m} O\left(\varepsilon^{\beta}\right) \rightarrow 0,
\end{aligned}
$$

where $\beta=N / 2 m-1 /(p-1)>0$. This gives the rate of convergence as $\varepsilon \rightarrow 0$ of $\left\{u_{\varepsilon}\right\}$ to the trivial proper solution in the supercritical range $p>p_{0}$,

$$
\bar{u}(t) \equiv \lim _{\varepsilon \rightarrow 0} u_{\varepsilon}(t)=0 \quad \text { for any } t>0 .
$$

Critical case $p=p_{0}$. Then $\gamma=0$ in (5.3) and we do not expect such a fast convergence to zero as in 5.11. We now use the fact that the PDE is invariant under a group of scaling transformations. Namely, if $u(x, t)$ is a solution of (1.6), then for any $\lambda>0$,

$$
u_{\lambda}(x, t)=\mathcal{T}_{\lambda} u \equiv \lambda^{-1 /(p-1)} u\left(x / \lambda^{1 / 2 m}, t / \lambda\right)
$$

is a solution. For $p=p_{0}$, we have $1 /(p-1)=N / 2 m$, so that for any $\varepsilon>0$,

$$
u_{\varepsilon}(x, t)=\mathcal{T}_{\varepsilon} U \equiv \varepsilon^{-N / 2 m} U\left(x / \varepsilon^{1 / 2 m}, t / \varepsilon\right),
$$

where $U=U(y, \tau)$ is a unique classical solution of the Cauchy problem

$$
U_{\tau}=-(-\Delta)^{m} U-|U|^{p-1} U \quad \text { for } \tau>0, \quad U(y, 0)=f(y) .
$$

It was shown in [8] that in the critical case $p=p_{0}$, for initial data in a weighted $L^{2}$-space, equation 5.15 admits global solutions with logarithmically perturbed asymptotics of the fundamental solution 2.2,

$$
U(y, \tau)=C_{*}(\tau \ln \tau)^{-N / 2 m}\left[f\left(y / \tau^{1 / 2 m}\right)+o(1)\right], \quad \tau \rightarrow \infty,
$$


where $C_{*}$ is a bounded constant (possibly zero). If the solution of the Cauchy problem (5.15) satisfies $\left(5.16\right.$ with $C_{*} \neq 0$, it then follows from 5.14 ) that the approximating sequence $\left\{u_{\varepsilon}\right\}$ has the following behaviour for a fixed small $t_{0}>0$ :

$$
u_{\varepsilon}\left(x, t_{0}\right)=t_{0}^{-N / 2 m} O\left(|\ln \varepsilon|^{-N / 2 m}\right) \rightarrow 0 \quad \text { as } \varepsilon \rightarrow 0 .
$$

For $m=1$ this is proved in [10, Sect. 6] for the porous medium equation with critical absorption. Thus we obtain a (slower than in (5.11)) logarithmic convergence of $\left\{u_{\varepsilon}\right\}$ to the trivial proper solution so that 5.12 also holds for $p=p_{0}$.

\subsection{Blow-up boundary layer in the reaction case}

We now consider equation (1.9) admitting blow-up solutions and identify proper solutions.

Supercritical range. For $p>p_{0}$, performing the same scaling (5.2), (5.7), we obtain the Cauchy problem for $\left\{v_{\varepsilon}\right\}$

$$
v_{s}=\psi_{\varepsilon}(s) \mathbf{B} v+|v|^{p} \quad \text { for } s>0, \quad v(y, 0)=f(y) .
$$

It follows from 5.9 that $\psi_{\varepsilon}(s) \rightarrow 0$ as $\varepsilon \rightarrow 0$ uniformly on any bounded interval in $s$. One can expect that the behaviour of $v(s)$ for $\varepsilon \approx 0$ is described by the ODE

$$
V_{s}=|V|^{p} \quad \text { for } s>0, \quad V(y, 0)=f(y) .
$$

Obviously, $V$ blows up. For instance, since $f(0)>0$ by 2.4), at the origin this happens at $s_{*}=f^{1-p}(0) /(p-1)$, and hence 5.7 implies that $u_{\varepsilon}(t)$ blows up at

$$
t_{\varepsilon *}=O\left(\varepsilon^{\gamma+1}\right) \rightarrow 0 \quad \text { as } \varepsilon \rightarrow 0 .
$$

Thus, the approximating solutions $\left\{u_{\varepsilon}\right\}$ blow up at small $t_{\varepsilon *}$ for any $\varepsilon \ll 1$ so that the proper solution via the limit as in 5.12 ) is not well defined. On the other hand, for secondorder equations, such a situation typically means that the proper solution and the extended limit semigroup can be defined in such a way and $\bar{u}(t)=\infty$ for any $t>0$ corresponding to complete blow-up at $t=0$ (see [11]). For higher-order equations, the problem on complete or incomplete finite-time singularities remains open (i.e., we do not know what happens to $u_{\varepsilon}(t)$ for $\left.t>t_{\varepsilon *}\right)$. Nevertheless, we expect that in the present situation $\bar{u}(t)=$ $\pm \infty$ (infinite oscillations are possible) is a "right" proper solution of the Cauchy problem.

Critical case. For $p=p_{0}$ we define the approximating sequence $\left\{u_{\varepsilon}\right\}$ according to (5.14), and this leads to the following problem for $U$ :

$$
U_{\tau}=-(-\Delta)^{m} U+|U|^{p} \quad \text { for } \tau>0, \quad U(y, 0)=f(y) .
$$

Since the initial function satisfies $\int f=1>0$, the solution $U(y, \tau)$ blows up in the $L^{\infty}$. norm at a finite time $\tau_{*}$ (see different proofs in [5] and [9]). Hence, each $u_{\varepsilon}(x, t)$ blows up at $t_{\varepsilon *}=\tau_{*} \varepsilon$ (cf. 5.20) for $\gamma=0$ ). As above, we expect that in this case $\bar{u}(t) \equiv \pm \infty$ is a proper solution created by this singularity formation phenomenon at $t=0$.

Acknowledgements. The author would like to thank H. Brezis for a stimulating discussion. 


\section{References}

[1] Wang, B.: The Cauchy problem for critical and subcritical semilinear parabolic equations in $L^{r}$ (I). Nonlinear Anal. 48, 747-764 (2002) Zbl 1002.35057 MR 2002i:35095

[2] Baras, P., Cohen, L.: Complete blow-up after $T_{\max }$ for the solution of a semilinear heat equation. J. Funct. Anal. 71, 142-174 (1987) Zbl 0653.35037 MR 88e:35105

[3] Brezis, H., Friedman, A.: Nonlinear parabolic equations involving measures as initial conditions. J. Math. Pures Appl. 62, 73-97 (1983) Zbl 0527.35043 MR 84g:35093

[4] Cui, S.: Local and global existence of solutions to semilinear parabolic initial value problems. Nonlinear Anal. 43, 293-323 (2001) Zbl 0963.35075 MR 2001m:35153

[5] Egorov, Yu. V., Galaktionov, V. A., Kondratiev, V. A., Pohozaev, S. I.: On the necessary conditions of existence to a quasilinear inequality in the half-space. C. R. Acad. Sci. Paris Sér. I 330, 93-98 (2000) Zbl 0943.35110 MR 2001b:35309

[6] Egorov, Yu. V., Galaktionov, V. A., Kondratiev, V. A., Pohozaev, S. I.: Asymptotic behaviour of global solutions to higher-order semilinear parabolic equations in the supercritical range. C. R. Acad. Sci. Paris Sér. I 335, 805-810 (2002) (full text at http://www.maths.bath.ac.uk/ MATHEMATICS/preprints.html) MR 1947704

[7] Eidel'man, S. D.: Parabolic Systems. North-Holland, Amsterdam (1969) Zbl 0181.37403 MR 40 \#6023

[8] Galaktionov, V. A.: Critical global asymptotics in higher-order semilinear parabolic equations. Int. J. Math. Math. Sci., to appear (http://www.maths.bath.ac.uk/MATHEMATICS/ preprints.html).

[9] Galaktionov,V. A., Pohozaev, S. I.: Existence and blow-up for higher-order semilinear parabolic equations: majorizing order-preserving operators. Indiana Univ. Math. J. 51, 13211338 (2002) MR 1948452

[10] Galaktionov, V. A., Vazquez, J. A.: Asymptotic behaviour of nonlinear parabolic equations with critical exponents. A dynamical systems approach. J. Funct. Anal. 100 (1991), 435-462 Zbl 0755.35010 MR 92k:35128

[11] Galaktionov, V. A., Vazquez, J. A.: Continuation of blowup solutions of nonlinear heat equations in several space dimensions. Comm. Pure Appl. Math. 50, 1-68 (1997) Zbl 0874.35057 MR 97h:35085

[12] Tanabe, H.: Functional Analytic Methods for Partial Differential Equations. Monogr. Textbooks Pure Appl. Math. 204, Dekker, New York (1997) Zbl 0867.35003 MR 97i:35002

[13] Taylor, M. E.: Partial Differential Equations III. Nonlinear Equations. Springer, New York (1996) Zbl 0869.35004 MR 98k:35001

[14] Weissler, F. B.: Semilinear evolution equations in Banach spaces. J. Funct. Anal. 32, 277-296 (1979) Zbl 0419.47031 MR 80i:47091

[15] Weissler, F. B.: Local existence and nonexistence for semilinear parabolic equations in $L^{p}$. Indiana Univ. Math. J. 29, 79-102 (1980) Zbl 0443.35034 MR 81c:35072 